\title{
On the Domain of Four Dimensional Pascal Matrix in the Space 1_q^2
}

\author{
Ahmadu Kiltho ${ }^{1}$, Jidda Bashir ${ }^{2}$ and A. M. Brono ${ }^{3}$ \\ ${ }^{1,2,3}$ Department of Mathematical Sciences, University of Maiduguri, Maiduguri \\ DOI: 10.29322/IJSRP.11.09.2021.p11739 \\ http://dx.doi.org/10.29322/IJSRP.11.09.2021.p11739
}

\begin{abstract}
We introduce the double sequence space p_q $\mathrm{q}^{\wedge} 2=\mathrm{P}\left(1 \_\mathrm{q}^{\wedge} 2\right.$ ) as the domain of four dimensional Pascal matrix $P$ in the space $1 \_q^{\wedge} 2$, for $1 \leq q<\infty$. Furthermore, we show that $p_{-} q^{\wedge} 2$ is a BKspace, Banach space, establish its Schauder basis, topological properties, isomorphism and some inclusions.
\end{abstract}

Index Terms- 4-dimensional Pascal Matrix, Isomorphism and inclusions

2018 MSC: 40C05, 40D25, 406A45, 406B03

\section{BASIC NOTATIONS AND BACKGROUND}

$\mathrm{L}$ et $\iota: \mathbb{N} \times \mathbb{N} \rightarrow \tau$ be a function, where $\tau$ may stand for any nonempty set and $\mathbb{N}$ a set of counting numbers. Then $(j, k) \rightarrow$ $\iota(j, k)=x_{j k}$ can be termed to be a double sequence.

In the paper by Basar $\&$ Sever [2] the Banach space $l_{q}^{2}$ of double sequences corresponding to the well-known $l_{q}$ of single sequences was introduced, its properties were studied, and its $\beta(v)$-dual determined and the established that the $\alpha$ - and $\delta$ duals of the space coincide with the $\beta(v)$-dual; where $1 \leq q<$ $\infty$ and $v \in\{p, b p, r\}$. If $P$ denotes the Pascal mean (a four dimensional matrix), then $p_{\infty}^{2}, p_{c}^{2}, p_{b c}^{2}$ and $p_{0}^{2}$ are collections of all double sequences whose $P$-transforms are in the spaces $l_{\infty}^{2}, c^{2}, c_{b}^{2}$ and $c_{0}^{2}$ respectively; where $l_{\infty}^{2}, c^{2}, c_{b}^{2}$ and $c_{0}^{2}$ are the double spaces of bounded, convergent, both bounded and convergent and null sequences respectively in the Pringsheim's sense, see Moricz [3]. We introduce a new double sequence space $p_{q}^{2}$ of Pascal as the set of all double sequences whose $P$ transforms are in the space $l_{q}^{2}$. Next, fixing some notations is necessary.

Let $\omega^{2}$ be a vector space of all real or complex valued double sequences for which coordinatewise addition and scalar multiplications are defined, see Moricz [3]. Further, a vector subspace of $\omega^{2}$ is termed as a double sequence space, see Moricz [3]. The space $l_{\infty}^{2}$ denotes the space of all bounded sequences with $\operatorname{norm}\|x\|_{\infty}=\sup _{j, k \in \mathbb{N}}\left|x_{j k}\right|<\infty, N=\{1,2,3, \ldots\} . \quad$ If $x=x_{j k} \in \mathbb{C}$, then $x$ is convergent to a number $l$ in Pringsheim's sense if for every $\varepsilon>0$, there exists a number $n_{0}=n_{0}(\varepsilon) \in \mathbb{N}$ and $l \in \mathbb{C}$ such that $\left|x_{j k}-l\right|<\varepsilon \forall j, k \geq n_{0}$, and we write $P-\lim _{j, k \rightarrow \infty} x_{j k}=l, \mathbb{C}$ being the complex field, see Pringsheim [4]. $c^{2}$ is used to denote the space of all convergent double sequences in Pringsheim's sense, see Moricz [3]. In Moricz [3], it is pointed out that there are some double sequences in $c^{2}$ that are not in $l_{\infty}^{2}$; for example, the double sequence $x=\left(x_{j k}\right)$ defined by

$$
x_{j k}:=\left\{\begin{array}{l}
n, j=0, k \in \mathbb{N}, \\
0, j \geq 1, k \in \mathbb{N} .
\end{array}\right.
$$

In Moricz [3], it can be seen that $x$ is convergent in Pringsheim's sense but not bounded, since $p-\lim _{j, k \rightarrow \infty} x_{j k}=0$ but $\|x\|_{\infty}=\infty$. Hence, we consider the space $c_{b}^{2}$ of all double sequences which are both convergent in Pringsheim's sense and bounded; that is, $c_{b}^{2}=$ $c^{2} \cap l_{\infty}^{2}$, Moricz [3]. So, $c_{0}^{2}$ is the space of all double sequences converging to zero in Pringsheim's sense, and $c_{0 b}^{2}$ is the space of all double sequences that are bounded and converging to zero in Pringsheim's sense, i.e., $c_{0 b}^{2}=c_{0}^{2} \cap l_{\infty}^{2}$, Moricz [3]. Also, Basar and Sever [2] defined the space $l_{q}^{2}$ by

$$
l_{q}^{2}:=\left\{x=\left(x_{j k}\right) \in \omega^{2}: \sum_{j, k=0,0}^{\infty, \infty}\left|x_{j k}\right|^{q}<\infty\right\},(1 \leq q<\infty) .
$$

For $1 \leq q<\infty$, Basar and Sever [2] proved that the double sequence space $l_{q}^{2}$, corresponds to $l_{q}$ of single sequences, and is a Banach space with the norm

$$
\begin{aligned}
& \|x\|_{l_{q}^{2}}:=\left(\sum_{j, k=0,0}^{\infty, \infty}\left|x_{j k}\right|^{q}\right)^{\frac{1}{q}}, \\
& 1 \leq q<\infty
\end{aligned}
$$

In Basar and Sever [2], in case $q \in(0,1)$ in (1), then the space $l_{q}^{2}$ is a $q$-norm with the $q$-norm

$$
\||x|\|:=\|x\|_{l_{q}^{2}}^{q}=\sum_{j, k=0,0}^{\infty, \infty}\left|x_{j k}\right|^{q} .
$$

Let $X$ and $Y$ be two double sequence spaces and $A=$ $\left(a_{m n j k}\right)$ be any four-dimensional infinite matrix of complex numbers as in Robison [16]. In Robison [16], it was also indicated that $A$ is said defines a matrix mapping from $X$ into $Y$ and write $A: X \rightarrow Y$ for every $x=\left(x_{j k}\right) \in X$, so that the $A$-transform of $x=\left(x_{j k}\right)$ is $A x=\left\{(A x)_{m n}\right\}_{m n}$, where

$(A x)_{m n}$
$=P$
$-\sum_{j, k}^{m, n} a_{m n j k} x_{j k}$

for each $m, n \in \mathbb{N}$, exists. For matrix domains, Yesilkayagil \& Basar [10], the $v$-matrix domain $\chi_{A}^{(v)}$ of $A$ in $X$ is defined by 


$$
\begin{aligned}
\chi_{A}^{(v)}=\left\{x=\left(x_{j k}\right)\right. & \\
& \left.\in \omega^{2}: P-\sum_{j, k}^{m, n} a_{m n j k} x_{j k} \text { exists and is in } Y\right\} .
\end{aligned}
$$

Clearly, (2) suggests that $A$ maps $X$ into $Y$ if $X \subset Y_{A}^{(v)}$; and $(X: Y)$ can denote the set of all four-dimensional matrices transforming $X$ into $Y$, see Yesilkayagil \& Basar [10]. $A=\left(a_{m n j k}\right) \in(X: Y)$ if, and only if the double series on the right of (1) converges in Pringsheim's sense for each $m, n \in \mathbb{N}$, that is, $A_{m n} \in \chi^{\beta(v)}$ for all $j, k \in \mathbb{N}$ and any $x \in X$ have $A x \in Y$, see Yesilkayagil \& Basar [10]. It is well known, for example in Cooke [15], that $A=$ $\left(a_{m n j k}\right)$ is a triangular matrix if $a_{m n j k}=0$ for $j>m, k>m$ or both, and $a_{m n j k} \neq 0$ for all $m, n \in \mathbb{N}$ and every triangular matrix has a unique inverse which also happens to be a triangular matrix too.

\section{The Pascal Double Sequence Space $\boldsymbol{p}_{\boldsymbol{q}}^{\boldsymbol{2}}$}

Pascal matrix of finite order existed for a very long time as pointed out by Aggarwala \& Lamoureux [5], where the authors declared that there was no reason, whatsoever, to stop at a finite matrix of this type for, one can extend the Pascal matrix of finite order to an infinite lower triangular matrix. We felt that this extension aroused Polat [1] to introduce some Pascal sequence spaces, each which is a matrix domain via infinite Pascal matrix as follows:

$$
\begin{gathered}
\left(l_{\infty}\right)_{P}=p_{\infty}=\left\{x=\left(x_{k}\right) \in \omega: \sup _{n}\left|\sum_{k}^{n}\left(\begin{array}{c}
n \\
n-k
\end{array}\right) x_{k}\right|<\infty\right\} \\
(c)_{P}=p_{c}=\left\{x=\left(x_{k}\right) \in \omega: \lim _{n \rightarrow \infty} \sum_{k}^{n}\left(\begin{array}{c}
n \\
n-k
\end{array}\right) x_{k} \text { exists }\right\} \\
\left(c_{0}\right)_{P}=p_{0}=\left\{x=\left(x_{k}\right) \in\right.
\end{gathered}
$$

$\left.\omega: \lim _{n \rightarrow \infty} \sum_{k}^{n}\left(\begin{array}{c}n \\ n-k\end{array}\right) x_{k}=0\right\}$.

Recently, Kiltho, et al. [17] introduced Pascal double sequence spaces, $p_{\infty}^{2}, p_{c}^{2}, p_{b c}^{2}$ and $p_{0}^{2}$ as matrix domains of four-dimensional Pascal matrix, as an extension of the work of Polat [1]. This paper will therefore wish to introduce the Pascal double sequence space $p_{q}^{2}$, the set of all double sequences whose $P$-transforms are in the space $l_{q}^{2}$. We define the four-dimensional Pascal matrix $P=$ $\left(p_{m n}^{j k}\right)$ as follows:

$p_{m n}^{j k}$

$=\left\{\begin{array}{cc}\left(\begin{array}{c}m \\ m-j\end{array}\right)\left(\begin{array}{c}n \\ n-k\end{array}\right), & 0 \leq j \leq m, 0 \leq k \leq n \\ 0 & , j>m \text { and } k>n .\end{array}\right.$

with inverse $P^{-1}=Q=\left(q_{m n}^{j k}\right)$ defined by

$=\left\{\begin{array}{c}q_{m n}^{j k}(-1)^{(m-j)+(n-k)}\left(\begin{array}{c}m \\ m-j\end{array}\right)\left(\begin{array}{c}n \\ n-k\end{array}\right), 0 \leq j \leq m, 0 \leq k \leq n \\ 0 \quad, j>m \text { and } k>n .\end{array}\right.$
Now, we introduce the space $p_{q}^{2}$ as the collection of all double sequences such that its $P$-transform is in the space $l_{q}^{2}$, as follows

$$
\left.\begin{array}{rl}
p_{q}^{2}=\left\{x=\left(x_{j k}\right)\right. & \in \omega^{2}: \sum_{m, n}\left|\sum_{j, k=0}^{m, n}\left(\begin{array}{c}
m \\
m-j
\end{array}\right)\left(\begin{array}{c}
n \\
n-k
\end{array}\right) x_{j k}\right|^{q} \\
& <\infty
\end{array}\right\}
$$

Following Yesilkayagil \& Basar [10] the space $p_{q}^{2}$ is linear with coordinatewise addition and scalar multiplication, where we are going to show that it is a complete $q$-normed space with the q-norm:

$\|x\|_{p_{q}^{2}}$
$=\left(\sum_{m, n}\left|\sum_{j, k=0}^{m, n}\left(\begin{array}{c}m \\ m-j\end{array}\right)\left(\begin{array}{c}n \\ n-k\end{array}\right) x_{j k}\right|^{q}\right)^{\frac{1}{q}}$.
Let $\chi_{A}=\left\{x=\left(x_{j k}\right) \in \omega^{2}: A x\right.$

Let $\chi_{A}=\left\{x=\left(x_{j k}\right) \in \omega^{2}: A x \in X\right\}$ be a matrix domain of a four-dimensional matrix $A$, then the Pascal sequence space in (6) is also a matrix domain, as $p_{q}^{2}=\left(l_{q}^{2}\right)_{P}=P\left(l_{q}^{2}\right)$; while $P$ transform of a double sequence space $x=\left(x_{j k}\right)$ in (6) can be defined as

$y_{m n}=(P x)_{m n}$
$=\sum_{j, k=0}^{m, n}\left(\begin{array}{c}m \\ m-j\end{array}\right)\left(\begin{array}{c}n \\ n-k\end{array}\right) x_{j k}$

for all $m, n \in \mathbb{N}$. The terms of the double series $x=\left(x_{m n}\right)$ and $y=\left(y_{m n}\right)$ are assumed to be connected with the relation (8).

Definition 1: A Banach space is called a $B K$-space provided each of the maps $p_{j k}: X \rightarrow \mathbb{C}$ defined by $p_{j k}=x_{j k}$ is continuous for all $j, k \in \mathbb{N}$, see Choudhary \& Nanda [12]

Lemma 1: If $A$ is a triangle and $X$ is a $B K$-space, then $X_{A}$ is a $B K$-space with the norm given by $\|x\|_{X_{A}}=\|x\|_{X}$ for all $x \in X_{A}$, see Boos [11].

By considering the notion of $B K$-space, one can say that the sequence space $l_{q}^{2}$ is a $B K$-space according to its $l_{q}^{2}$-normed defined by $\|x\|_{l_{q}^{2}}=\left(\sum_{m, n=0,0}^{\infty, \infty}\left|x_{j k}\right|^{q}\right)^{\frac{1}{q}}$, where $1 \leq q<\infty$. Next, we present our results viz:

Theorem 1: The Pascal sequence $p_{q}^{2}$ is a $B K$-space according to the norm defined by

$$
\|x\|_{p_{q}^{2}}=\|P x\|_{l_{q}^{2}}=
$$

$\left(\sum_{m, n}\left|\sum_{j, k=0}^{m, n}\left(\begin{array}{c}m \\ m-j\end{array}\right)\left(\begin{array}{c}n \\ n-k\end{array}\right) x_{j k}\right|^{q}\right)^{\frac{1}{q}}$, where $1 \leq q<\infty$ as in (7).

Proof: Since $l_{q}^{2}$ is a $B K$ space, we define a map $\mathrm{A}_{P}: p_{q}^{2} \rightarrow l_{q}^{2}$ by $\mathrm{A}_{P}(x)=P(x) \forall x \in p_{q}^{2}$. Since $P$ is a triangular matrix, then $A_{P}$ is linear, 0ne-to-one and onto. If $A_{P}^{-1}$ is the inverse of $A_{P}$, it is also linear, one-to-one and onto, so that $p_{q}^{2}=\mathrm{A}_{P}^{-1}\left(l_{q}^{2}\right)$ is a Banach space. It remains to show the coordinates are continuous in $l_{q}^{2}$. To do this, let $x_{j k} \rightarrow x$ in $p_{q}^{2}$. Then $y_{j k}^{[r, s]}=P\left(x^{[r, s]}\right) \Rightarrow$ $y_{j k}=P(x)_{5}$, since $l_{q}^{2}$ is a $B K$ space. Let $P^{-1}=Q$ be the inverse of $P$, which is also a triangle. Then $x_{m n}^{[r, s]}=\sum_{j, k=0}^{m, n} Q y_{m n}^{[r, s]} \rightarrow$ 
$\sum_{j, k=0}^{m, n} Q y_{m n}=x$. This shows that the coordinates are continuous on $p_{q}^{2}$. Hence $p_{q}^{2}$ is a $B K$ space.

Theorem 2: The set $p_{q}^{2}$ becomes a linear space with the coordinatewise addition and scalar multiplication and the following statements hold:

i) If $q \in(0,1)$, then $p_{q}^{2}$ is a complete $q$-normed space with

$$
\|x\|_{p_{q}^{2}}=\sum_{m, n}\left|\sum_{j, k=0}^{m, n}\left(\begin{array}{c}
m \\
m-j
\end{array}\right)\left(\begin{array}{c}
n \\
n-k
\end{array}\right) x_{j k}\right|^{q}
$$

which is $q$-norm isomorphic to the space $l_{q}^{2}$.

ii) If $q \in[1, \infty) \mathrm{q} \in 1, \infty$ then $p_{q}^{2}$ is a Banach space with

$$
\|x\|_{p_{q}^{2}}=\left(\sum_{m, n}\left|\sum_{j, k=0}^{m, n}\left(\begin{array}{c}
m \\
m-j
\end{array}\right)\left(\begin{array}{c}
n \\
n-k
\end{array}\right) x_{j k}\right|^{q}\right)^{\frac{1}{q}}
$$

which is isomorphic to the space $l_{q}^{2}$.

Proof: We are going to give the proof of the second part (ii), since the first part (i) can be proved in a similar way.

The first part of the theorem is a routine verification, where it can be easily seen that (a) $p_{q}^{2}$ is not empty; (b) the sum of any two elements in $p_{q}^{2}$ is also in $p_{q}^{2}$; and (c) the scalar multiplication $\alpha x \in p_{q}^{2} \forall \alpha \in \mathbb{C}$ and $x \in p_{q}^{2}$. Thus, $p_{q}^{2}$ is a linear space with coordinatewise addition and scalar multiplication. Now, we can show that $p_{q}^{2}$ is a Banach space with the norm defined by (7). Let $\left(x^{\alpha}\right)_{\alpha \in \mathbb{N}}$ be any Cauchy sequence in the space $p_{q}^{2}$, where $x^{\alpha}=$ $\left\{x_{j k}{ }^{(\alpha)}\right\}_{j, k=0}^{\infty}$ for every fixed $\alpha \in \mathbb{N}$. Then for a given $\varepsilon>0$, there exists a positive integer $N=N(\varepsilon)$ such that

$$
\begin{aligned}
\left\|x^{\alpha}-x^{\beta}\right\|_{p_{q}^{2}}= & \left(\sum_{m, n}\left|\sum_{j, k=0}^{m, n}\left(\begin{array}{c}
m \\
m-j
\end{array}\right)\left(\begin{array}{c}
n \\
n-k
\end{array}\right)\left(x_{j k}^{\alpha}-x_{j k}^{\beta}\right)\right|^{q}\right)^{\frac{1}{q}} \\
& <\varepsilon \quad \forall \alpha, \beta>N
\end{aligned}
$$

which yields for each $m, n \in \mathbb{N}$ and applying Minkowki's inequality, that

$$
\begin{aligned}
& \left\|x^{\alpha}-x^{\beta}\right\|_{p_{q}^{2}}=\left(\sum_{m, n} \mid \sum_{j, k=0}^{m, n}\left(\begin{array}{c}
m \\
m-j
\end{array}\right)\left(\begin{array}{c}
n \\
n-k
\end{array}\right) x_{j k}^{\alpha}\right. \\
& \left.-\left.\sum_{j, k=0}^{m, n}\left(\begin{array}{c}
m \\
m-j
\end{array}\right)\left(\begin{array}{c}
n \\
n-k
\end{array}\right) x_{j k}^{\beta}\right|^{q}\right)^{\frac{1}{q}} \\
& \leq \mid\left(\sum_{m, n}\left|\sum_{j, k=0}^{m, n}\left(\begin{array}{c}
m \\
m-j
\end{array}\right)\left(\begin{array}{c}
n \\
n-k
\end{array}\right) x_{j k}^{\alpha}\right|^{q}\right)^{\frac{1}{q}} \\
& -\left(\left|\sum_{j, k=0}^{m, n}\left(\begin{array}{c}
m \\
m-j
\end{array}\right)\left(\begin{array}{c}
n \\
n-k
\end{array}\right) x_{j k}^{\beta}\right|^{q}\right)^{\frac{1}{q}} \mid<\varepsilon
\end{aligned}
$$

This means that $\left(\sum_{m, n}\left|\sum_{j, k=0}^{m, n}\left(\begin{array}{c}m \\ m-j\end{array}\right)\left(\begin{array}{c}n \\ n-k\end{array}\right) x_{j k}^{\alpha}\right|^{q}\right)^{\frac{1}{q}}{ }_{\alpha \in \mathbb{N}}$ is a Cauchy sequence with complex terms for every fixed $m, n \in \mathbb{N}$. Since $\mathbb{C}$ is complete, it converges, i.e.

$$
\begin{aligned}
\left(\sum_{m, n} \mid \sum_{j, k=0}^{m, n}\left(\begin{array}{c}
m \\
m-j
\end{array}\right)\right. & \left.\left.\left(\begin{array}{c}
n \\
n-k
\end{array}\right) x_{j k}^{\alpha}\right|^{q}\right)^{\frac{1}{q}} \\
& \rightarrow\left(\sum_{m, n}\left|\sum_{j, k=0}^{m, n}\left(\begin{array}{c}
m \\
m-j
\end{array}\right)\left(\begin{array}{c}
n \\
n-k
\end{array}\right) x_{j k}\right|^{q}\right)^{\frac{1}{q}}
\end{aligned}
$$

$\infty$,

such that

$$
\lim _{\alpha \rightarrow \infty}\left\|x^{\alpha}-x^{\beta}\right\|_{p_{q}^{2}}=0 .
$$

Since, $\left(\sum_{m, n}\left|\sum_{j, k=0}^{m, n}\left(\begin{array}{c}m \\ m-j\end{array}\right)\left(\begin{array}{c}n \\ n-k\end{array}\right) x_{j k}^{\alpha}\right|^{q}\right)^{\frac{1}{q}}{ }_{m, n \in \mathbb{N}} \in p_{q}^{2}$ for each fixed $\alpha \in \mathbb{N}$, there exists a positive real number $K_{\alpha}$ such that

$$
\left(\sum_{m, n}\left|\sum_{j, k=0}^{m, n}\left(\begin{array}{c}
m \\
m-j
\end{array}\right)\left(\begin{array}{c}
n \\
n-k
\end{array}\right) x_{j k}^{\alpha}\right|^{q}\right)^{\frac{1}{q}} \leq K_{\alpha} .
$$

Therefore, taking summation over $m, n$ in the following relation

$$
\begin{gathered}
\left|\sum_{j, k=0}^{m, n}\left(\begin{array}{c}
m \\
m-j
\end{array}\right)\left(\begin{array}{c}
n \\
n-k
\end{array}\right) x_{j k}\right|^{\frac{1}{q}} \\
=\mid \sum_{j, k=0}^{m, n}\left(\begin{array}{c}
m \\
m-j
\end{array}\right)\left(\begin{array}{c}
n \\
n-k
\end{array}\right) x_{j k}-\sum_{j, k=0}^{m, n}\left(\begin{array}{c}
m \\
m-j
\end{array}\right)\left(\begin{array}{c}
n \\
n-k
\end{array}\right) x_{j k}^{\alpha} \\
+\left.\sum_{j, k=0}^{m, n}\left(\begin{array}{c}
m \\
m-j
\end{array}\right)\left(\begin{array}{c}
n \\
n-k
\end{array}\right) x_{j k}^{\alpha}\right|^{\frac{1}{q}} \\
\leq\left|\sum_{j, k=0}^{m, n}\left(\begin{array}{c}
m \\
m-j
\end{array}\right)\left(\begin{array}{c}
n \\
n-k
\end{array}\right) x_{j k}-\sum_{j, k=0}^{m, n}\left(\begin{array}{c}
m \\
m-j
\end{array}\right)\left(\begin{array}{c}
n \\
n-k
\end{array}\right) x_{j k}^{\alpha}\right|^{\frac{1}{q}} \\
+\left|\sum_{j, k=0}^{m, n}\left(\begin{array}{c}
m \\
m-j
\end{array}\right)\left(\begin{array}{c}
n \\
n-k
\end{array}\right) x_{j k}^{\alpha}\right|^{\frac{1}{q}}
\end{gathered}
$$$$
\leq \varepsilon+K_{\alpha} .
$$

This shows that $x=\left(x_{j k}\right) \in p_{q}^{2}$. Since $\left\{x^{\alpha}\right\}_{\alpha \in \mathbb{N}}$ is an arbitrary Cauchy sequence, then the space $p_{q}^{2}$ is complete. Thus, $p_{q}^{2}$ is a Banach space with the norm $\|x\|_{p_{q}^{2}}=\left(\sum_{\mathrm{m}, \mathrm{n}}\left|(P x)_{m n}\right|^{q}\right)^{\frac{1}{q}}$.

To prove the fact that $p_{q}^{2}$ is linearly isomorphic to $l_{q}^{2}$, we have to show the existence of a linear bijection between the spaces $p_{q}^{2}$ and $l_{q}^{2}$. Consider the transformation $\tau$ defined from $p_{q}^{2}$ to $l_{q}^{2}$ by $x \mapsto y=\tau x=\left\{(P x)_{m n}\right\} \mathrm{y}=\tau x$. Clearly, $\tau$ is linear, $\tau(u)+\tau(v)=\tau(u+v) \tau u+\tau v=\tau(u+v)$ for all $u=\left(u_{j k}\right), v=$ $\left(v_{j k}\right) \in p_{q}^{2}$; and $K \cdot \tau(x)=\tau(K x)$ for all $K \in \mathbb{C}, x=\left(x_{j k}\right) \in p_{q}^{2}$. Further, we can see that $x=\theta$, whenever $\tau x=\theta$ which shows that $\tau$ is injective. Now, let $y=\left(y_{j k}\right) \in l_{q}^{2}$ and define a sequence $x=\left(x_{j k}\right)$ via $y$ by

$$
x_{j k}=\sum_{u, v=0}^{j, k}(-1)^{(j-u)+(k-v)}\left(\begin{array}{c}
j \\
j-u
\end{array}\right)\left(\begin{array}{c}
k \\
k-v
\end{array}\right) y_{u v} \quad \forall u, v \in \mathbb{N} .
$$


Hence, by taking into account the hypothesis $y \in l_{q}^{2}$, one can derive by taking summation over $m, n \in \mathbb{N}$ on the following equality $=\left|\sum_{j, k=0}^{m, n}\left(\begin{array}{c}m \\ m-j\end{array}\right)\left(\begin{array}{c}n \\ n-k\end{array}\right) \sum_{u, v=0}(P x)_{m n}\right|$
$=\left|y_{m n}\right|$.

That is, $\|P x\|_{q}=\|y\|_{q}$, which implies that $x \in p_{q}^{2}$. Therefore, $\tau$ is surjective. Hence, $p_{q}^{2} \cong l_{q}^{2}$.

Definition 2: A barreled space is a topological vector space for which every barreled set in the space is a neighbourhood for the zero vector. A barreled set in a topological vector space is a set that is convex, balanced, absorbing, and closed, see [Narici \& Beckenstein [13]

Lemma 2: If the sequence space $X$ is a Banach space or a Frechet space, then it is a barreled space, see Schaefer [14].

Theorem 3: The double sequence space $l_{q}^{2}$ is a barreled space for $1 \leq q<\infty$.

Proof: We have seen that $l_{q}^{2}$ is a Banach space in Theorem 2. Thus, the proof of Theorem 3 is obvious by Theorem 2 and Lemma 2.

Definition 3:The space $X$ of double sequence spaces is monotone if $x u=\left(x_{j k} u_{j k}\right) \in X$ fore very $x=\left(x_{j k}\right) \in X$ and $u=\left(u_{j k}\right) \in$ $\chi^{2}$, where $\chi^{2}=\{0,1\}^{\mathbb{N} \times \mathbb{N}}$ denotes the double sequence space of $0 \mathrm{~s}$ and 1s, see Yesilkayagil \& Basar [6].

Theorem 4: The space $l_{q}^{2}$ is monotone for all $1 \leq q<\infty$.

Proof: Let $1 \leq q<\infty, x=\left(x_{j k}\right) \in l_{q}^{2}$ and $u=\left(u_{j k}\right) \in \chi^{2}$. Then we have $\left|x_{j k} u_{j k}\right|^{q}=\left|x_{j k}\right|^{q}\left|u_{j k}\right|^{q} \leq\left|x_{j k}\right|^{q}$ for each $j, k \in \mathbb{N}$. This simply means that the inequality $\sum_{j, k}\left|x_{j k} u_{j k}\right|^{q} \leq \sum_{j, k}\left|x_{j k}\right|^{q}$ holds. That is, $x u=\left(x_{j k} u_{j k}\right) \in l_{q}^{2}$. This shows that $l_{q}^{2}$ is monotone for all $1 \leq q<\infty$.

Definition 4A double sequence $\left(x_{j k}\right)_{j, k=0}^{\infty}$ in a double sequence space $X$ is called a Schauder double basis if, for every $x \in X$, there exists a unique double sequence of scalars $\left(\lambda_{j k}\right)_{j, k=0}^{\infty}$ such that $x=\sum_{j, k=0}^{\infty} \lambda_{j k} x_{j k}$, see Loganathan \& Moorthy [8].

Theorem 5: Let $m, n, j, k \in \mathbb{N}$ and define $q^{(j k)}=\left\{q^{(j k)}\right\}_{m n}$ by $q_{m n}^{j k}$

$=\left\{\begin{array}{l}0 \\ (-1)^{(m-j)+(n-k)}\left(\begin{array}{c}m \\ m-j\end{array}\right)\left(\begin{array}{c}n \\ n-k\end{array}\right), 0 \leq k \leq n \text { and } k>n \text { or both, } 0 \leq j \leq m .\end{array}\right.$

Then the set $\left\{q^{(j k)}\right\}$ is a double basis for the double sequence space $p_{q}^{2}$ such that any $x \in p_{q}^{2}$ has a unique representation of the form

$$
\begin{aligned}
& x \\
& =\sum_{j k} \zeta_{j k} q^{(j k)} ; \\
& \text { where } \zeta_{j k}=(P x)_{j k} \forall j, k \in \mathbb{N} \text {. }
\end{aligned}
$$

Proof:

a) We want to show that $\left\{q^{(j k)}\right\} \subset p_{q}^{2}$. Since $P q^{j k}=e^{j k} \in$ $l_{q}^{2}$ for $j, k=0,1,2, \ldots \mathrm{k}=0,1,2, \ldots e^{j k}$ is a double sequence whose non-zero term is 1 in the $(j, k)^{\text {th }}$ place for each $j, k \mathrm{k}$ Now, let $x \in p_{q}^{2}$. For every $\mathrm{r}$ and $\mathrm{s}$, we write $x^{[r, s]}=$

$\sum_{j, k=0}^{r, s} \zeta_{j k} P q^{(j k)}$

$P$ is continuous. So, we can apply $P$ to $(10)$ to have

$$
\begin{aligned}
& x^{[r, s]}=\sum_{j, k=0}^{r, s} \zeta_{j k} P q^{(j k)}=x^{[r, s]} \\
& =\sum_{j, k=0}^{r, s}(P x)_{j k} e^{j k}
\end{aligned}
$$

and $\left\{P\left(x-x^{[r, s]}\right)\right\}_{i t}=\left\{\begin{array}{l}0,0 \leq i \leq r \& 0 \leq t \leq s \\ (P x)_{i t}, i>r \& t>s\end{array}\right.$. Let $\varepsilon>0$ be given. Then there exist $r_{0}$ and $s_{0}$ such that $\left|(P x)_{r, s}\right|<\frac{\varepsilon}{2} \forall r>$ $r_{0}$ and $s>s_{0}$. Therefore,

$$
\begin{aligned}
\left\|x-x^{[r, s]}\right\|_{p_{q}^{2}}= & \left(\sum_{i, t>r, s}\left|(P x)_{j k}\right|^{q}\right)^{\frac{1}{q}} \leq\left(\sum_{m, n>r_{0}, s_{0}}\left|(P x)_{j k}\right|^{q}\right)^{\frac{1}{q}} \\
& \leq \frac{\varepsilon}{2}<\varepsilon
\end{aligned}
$$

$\forall r>r_{0}$ and $s>s_{0}$. Clearly, this shows that $x \in p_{q}^{2}$ as in (10).

Next, we show the uniqueness of the representation of $x$ in (10). For this, suppose on the contrary that there exists another representation of $x=\sum_{j k} \xi_{j k} q^{(j k)}$. The linear transformation $P: p_{q}^{2} \rightarrow l_{q}^{2}$ is continuous. It implies that we can have

$$
\begin{gathered}
(P(x))_{m n}=\sum_{j, k} \xi_{j k}\left(P q^{(j k)}\right)_{m n}=\sum_{j, k} \xi_{j k} e^{j k} \\
=\xi_{j k} \quad(j, k \in \mathbb{N}),
\end{gathered}
$$

which is a clear contradiction to the fact that $(P(x))_{m n}=\zeta_{j k}$. So, the representation (10) is unique. This completes the proof.

Theorem 6: The inclusion $l_{q}^{2} \subset p_{q}^{2}$ holds for $1 \leq q<\infty$.

Proof: Let us take arbitrary $x=\left(x_{j k}\right) \in l_{q}^{2}$. Then there exists a positive real number $K$ Ksuch that $\sum_{j, k}\left|x_{j k}\right|^{q} \leq K$. Applying Holder's inequality to (8), we have

$$
\begin{gathered}
\left|y_{m n}\right|^{q}=\left|\sum_{j, k=0}^{m, n}\left(\begin{array}{c}
m \\
m-j
\end{array}\right)\left(\begin{array}{c}
n \\
n-k
\end{array}\right) x_{j k}\right|^{q} \\
\leq\left(\sum_{j, k=0}^{m, n}\left(\begin{array}{c}
m \\
m-j
\end{array}\right)\left(\begin{array}{c}
n \\
n-k
\end{array}\right)\left|x_{j k}\right|^{q}\right) \\
\times\left(\sum_{j, k=0}^{m, n}\left(\begin{array}{c}
m \\
m-j
\end{array}\right)\left(\begin{array}{c}
n \\
n-k
\end{array}\right)\right)^{q-1} \\
\leq \sum_{j, k=0}^{m, n}\left(\begin{array}{c}
m \\
m-j
\end{array}\right)\left(\begin{array}{c}
n \\
n-k
\end{array}\right)\left|x_{j k}\right|^{q}
\end{gathered}
$$

This implies that

$$
\sum_{m, n=0,0}^{\infty, \infty}\left|y_{m n}\right|^{q} \leq \sum_{m, n=0,0}^{\infty, \infty}\left(\sum_{j, k=0}^{m, n}\left(\begin{array}{c}
m \\
m-j
\end{array}\right)\left(\begin{array}{c}
n \\
n-k
\end{array}\right)\left|x_{j k}\right|^{q}\right)
$$




$$
\begin{gathered}
=\sum_{j, k=0}^{m, n}\left|x_{j k}\right|^{q}\left(\sum_{m, n=0,0}^{\infty, \infty}\left(\begin{array}{c}
m \\
m-j
\end{array}\right)\left(\begin{array}{c}
n \\
n-k
\end{array}\right)\right) \\
\sum_{j, k=0}^{m, n}\left|x_{j k}\right|^{q}\left(\sum_{m=0}^{\infty}\left(\begin{array}{c}
m \\
m-j
\end{array}\right) \sum_{n=0}^{\infty}\left(\begin{array}{c}
n \\
n-k
\end{array}\right)\right) \leq K
\end{gathered}
$$

That shows that $x=\left(x_{j k}\right) \in p_{q}^{2}$ for all $1 \leq q<\infty<\infty$ Hence, $l_{q}^{2} \subset$ $p_{q}^{2}$ holds for $1 \leq q<\infty$.

\section{REFERENCES}

[1] [1] Polat, H. (2018). Some new Pascal sequence spaces. Fundamental Journal of Mathematics and Applications, 1(1), 61-68.

[2] [2] Basar, F., \& Sever, Y. (2009). The space L_q of double sequences. Mathematical Journal of Okayama University, 51(1), 149-157.

[3] [3] Moricz, F. (1991). Extensions of the spaces c and c_0 from single to double sequences. Acta Mathematica Hungarica, 57(1-2), 129-136.

[4] [4] Pringsheim, A. (1900). Zur theorie der zweifach unendlichen Zahlenfolgen. Mathematische Annalen, 53(3), 289-321.

[5] [5] Aggarwala, R., \& Lamoureux, M. P. (2002). Inverting the Pascal matrix plus one. The American mathematical monthly, 109(4), 371-377.

[6] [6] Yesilkayagil, M., \& Basar, F. (2018). Domain of Euler mean in the space of absolutely $\mathrm{p}$-summable double sequences with $0<\mathrm{p}<1$. Anal. Theory Appl., 34(3), 241-252.

[7]

[8] [8] Loganathan, S., \& Moorthy, C. G. (2016). A net convergence for Schauder double bases. Asian-European Journal of Mathematics, 9(01), 1650010 .
[9] [9] Rao, K. C., \& Subramanian, N. (2004). The Orlicz space of entire sequences. International Journal of Mathematics and Mathematical Sciences, 2004(68), 3755-3764.

[10] [10] Yeşilkayagil, M., \& Başar, F. (2016). Some topological properties of the spaces of almost null and almost convergent double sequences. Turkish Journal of Mathematics, 40(3), 624-630.

[11] [11] Boos, J., \& Cass, F. P. (2000). Classical and modern methods in summability. Clarendon Press.

[12] [12] B. Choudhary and S. Nanda, Functional Analysis with Applications, Wiley, New Delhi (1989).

[13] [13] Beckenstein, E., Narici, L., \& Suffel, C. (2011). Topological algebras. Elsevier.

[14] [14] Schaefer, H. H. (1986). "On theorems of de Pagter and Ando-Krieger", Mathematische Zeitschrift, 192(1), 155-157.

[15] [15] Cooke, R G., (1950), "Sequence spaces and infinite matrices", Cambridge University Press, London

[16] [16] Robison, G M., (1926), "Divergent Double Sequences and Series", Trans. Amer. Math. Soc., 28, p. 50-73

[17] [17] Kiltho, A., Jidda Bashir and A. M. Brono, (2021), "Some sequence spaces generated by Four-Dimensional Pascal Matrix", Int. J. Math. \& Comp. Research, vol. 09 (08), p. 2371-2375

\section{AUTHORS}

First Author - Ahmadu Kiltho, Department of Mathematical Sciences, University of Maiduguri, Maiduguri

Second Author - Jidda Bashir, Department of Mathematical Sciences, University of Maiduguri, Maiduguri

Third Author - A. M. Brono, Department of Mathematical Sciences, University of Maiduguri, Maiduguri 